\title{
Advanced Photon Source Insertion Device Field Quality and Multipole Error
}

\section{Specification*}

\author{
Yong-Chul Chae and Glenn Decker \\ Argonne National Laboratory, 9700 So. Cass Ave., Argonne, Illinois 60439, U.S.A.
}

\section{Abstract}

The Advanced Photon Source (APS) storage ring is a 7-GeV light source with forty straight sections. Intense $x$ ray beams will be delivered by insertion devices installed in these straight sections. Installation of insertion devices in the APS storage ring produces several effects which can degrade overall performance. Rigid ring performance requirements exist which can be used to set limits on insertion device field quality, i.e. the first- and second-field integrals of the transverse magnetic field. Individual multipole error specifications can be determined by considering the lifetime of the beam. For nominal operation of the APS storage ring, the vertical aperture corresponding to a 10hour lifetime is approximately $3.35 \mathrm{~mm}$, which limits the level of multipole error. We find that the skew-octupole error has the most significant effect on the reduction of the aperture; the reasons are discussed in this paper.

\section{INTRODUCTION}

Installation of insertion devices (IDs) into the APS storage ring produces several effects which can degrade overall performance. Rigid ring performance requirements already exist which can be used to set limits on insertion device field quality. Shown in Table 1 is a list of insertion device properties and the storage ring parameters affected.

Table 1

Ring Performance Parameters Affected by Insertion Device Field Quality.

\begin{tabular}{|c|c|}
\hline Insertion Device Property & Ring Parameter Affected \\
\hline Field Integral, & Horizontal/Vertical Beam \\
$\mathrm{I}_{y, x}=\int B_{y, x} d l$ & Position Stability \\
\hline Second Field Integral, & Horizontal/Vertical Beam \\
$\mathrm{I} 2_{y, x}=\iint B_{y, x} d l d l^{\prime}$ & Position Stability \\
\hline Quadrupole Integral, & Tune, \\
$\int d B_{y} / d x d l$ & Beam Size \\
\hline Skew Quadrupole Integral, & Coupling, \\
$\int d B_{x} / d x d l$ & Beam Size \\
\hline Sextupole Integral, & Dynamic Aperture, \\
$\int d^{2} B / d x^{2} d l$ & Lifetime \\
\hline Octupole Integral, & Dynamic Aperture, \\
$\int d^{3} B / d x^{3}$ & Lifetime \\
\hline
\end{tabular}

-Work supported by U.S. Department of Energy, Office of Basic Energy Sciences under Contract No. W-31-109-ENG-38. GH
It is important to take into account the anticipated manner of operation of the insertion devices once they are installed in the ring, specifically the expectation that gaps will be scanned during operation. This places significantly tighter constraints on field quality than for the fixed gap operation, and dictates to a certain extent how the field quality specification should be written. The requirement that the interaction between a given insertion device and other beamlines be consistent with beam stability criterion limits the allowable field errors in that device. The maximum allowable changes in beam properties caused by insertion device operation place the strictest requirements on field quality.

\section{Effects of an Ideal Insertion Device}

Since in an ideal insertion device (ID) the first- and second-field integrals vanish, the device does not distort the equilibrium orbit. However it is well known that IDs have a vertical focusing effect on the beam. This can be illustrated by writing the Hamiltonian with respect to the oscillating equilibrium orbit suggested by L. Smith [1] as

$$
\begin{aligned}
H= & \frac{1}{2}\left(p_{x}^{2}+p_{y}^{2}\right)+\frac{1}{2}\left(\frac{1}{2 \rho^{2}}-\frac{p_{x} k}{\rho} \sin k s\right) y^{2} \\
& +\frac{k^{2}}{12 \rho^{2}} y^{4}+\frac{k^{4}}{90 \rho^{2}} y^{6}+\cdots,
\end{aligned}
$$

where $k=2 \pi / \lambda_{w}, \lambda_{w}$ is the period length, and $\rho$ is the radius of curvature in the ID peak field. If we neglect the fast oscillating (compared to betatron oscillation) term contained in $\sin k s$, we may treat the ID as a long quadrupole with all nonlinear elements lumped at the center. The tracking studies [2] based on this model showed identical results from the full-blown numerical integration through the ID field. The same study also showed that the ultimate dynamic aperture is limited by the nonlinear effects of the ID.

For the Type-A undulator which is one of the major IDs installed in APS storage ring, the effective integrated focusing strength is equal to 242 Gauss, which is two orders of magnitude smaller than the typical quadrupole strength in the ring. Thus, the perturbation of linear lattice functions due to the ID is negligible. However, the effective integrated octupole strength is equal to $600 \mathrm{Gauss} / \mathrm{cm}^{2}$, which has a serious effects on the dynamic aperture. In the specification of multipole tolerances of ID, the allowable multipole components will be compared with these values.

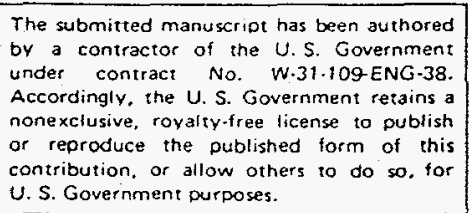




\section{DISCLAIMER}

This report was prepared as an account of work sponsored by an agency of the United States Government. Neither the United States Government nor any agency thereof, nor any of their employees, makes any warranty, express or implied, or assumes any legal liability or responsibility for the accuracy, completeness, or usefulness of any information, apparatus, product, or process disclosed, or represents that its use would not infringe privately owned rights. Reference herein to any specific commercial product, process, or service by trade name, trademark, manufacturer, or otherwise does not necessarily constitute or imply its endorsement, recommendation, or favoring by the United States Government or any agency thereof. The views and opinions of authors expressed herein do not necessarily state or reflect those of the United States Government or any agency thereof. 


\section{DISCLAIMER}

Portions of this document may be illegible in electronic image products. Images are produced from the best available original document. 


\section{Field Integral Specifications}

\section{A. Beam Position Stability}

The fundamental beam performance requirement from which ID field quality specifications can be inferred is beam position stability. This has been stated for the APS storage ring as shown in Table 2.

Table 2

APS Storage Ring Beam Stability Requirements

\begin{tabular}{|c|c|c|c|}
\hline$\Delta x$ & $\Delta x^{\prime}$ & $\Delta y$ & $\Delta y^{\prime}$ \\
\hline $16 \mu \mathrm{m}$ & $1.2 \mu \mathrm{rad}$ & $4.4 \mu \mathrm{m}$ & $0.45 \mu \mathrm{rad}$ \\
\hline
\end{tabular}

These requirements are derived from the need for the beam to be stable to within $10 \%$ of its emittance, which translates to $5 \%$ of its $\mathrm{rms}$ size. Table 2 assumes $\beta_{x}=14 \mathrm{~m}$ and $\beta_{y}=10 \mathrm{~m}$, which are the values at the IDs.

It is important that these stability specifications be interpreted as the maximum amount that the beam position can change with time. For example, it is acceptable to install a device which has a relatively large field integral, provided it does not change with time. If it does change appreciably with time, for example when scanning the gap, several things can still be done to stabilize the beam. First, because the amount of field integral change with gap is approximately known, one can introduce local steering compensation which tracks with gap, producing a net field integral which is independent of gap to the first order. This type of stabilization might be called "open loop" compensation.

It would be imprudent to assume that the open loop compensation of ID steering can be done significantly better than a factor of ten. Therefore, to generate an ID field integral specification guaranteed to meet beam stability requirements without being overly restrictive, the following procedure is proposed. First, compute the amount of field integral for one insertion device which will move the beam around the ring at most by the amounts in Table 2 . If we allow the uncompensated field integral to be ten times this amount, stability requirements can be met since it is safe to place a requirement of a factor of ten reduction from open loop compensation.

In fact, the APS storage ring will not have one ID, but rather thirty-four IDs. Because the contributions of these devices to the overall orbit motion is quasi-random, one would expect the net orbit motion to be on the order of $\sqrt{34}$ multiplied by the effect of a single ID. To compensate for this, we will rely on the real-time closed orbit feedback system using the photon beam position monitors. Since $\sqrt{34}$ is less than ten and we can assume that the feedback system will reduce the orbit distortion by a factor of ten, the requirement still can be met. Also, it is unlikely that all 34 beamlines will scan their gaps simultaneously, improving the situation further.

Of all the quantities in Table 2 , the restriction on vertical beam motion forms the tightest constraint. Specifi- cally, a change in insertion device field integral, gap open vs. gap closed, of $11.2 \mathrm{Gauss- \textrm {cm }}\left(\mathrm{I1}_{\mathrm{x}}\right.$ ) will produce $4.4 \mu \mathrm{m}$ of vertical beam motion somewhere around the ring. Horizontally, one arrives at 26.5 Gauss- $\mathrm{cm}$ of $\mathrm{II}_{y}$ to produce $16 \mu \mathrm{m}$ of motion. A conservative specification for the uncompensated field integral, as described above, is therefore 100 Gauss-cm.

Similar arguments for the second field integral indicate that 11,200 Gauss- $\mathrm{cm}^{2}$ of $12_{x}$ will produce $4.4 \mu \mathrm{m}$ of vertical beam motion. Also, 37,400 Gauss- $\mathrm{cm}^{2}$ of $12 y$ produces $16 \mu \mathrm{m}$ of horizontal beam motion. A reasonable requirement for the uncompensated second field integral is therefore 100,000 Gauss-cm ${ }^{2}$, or 0.001 Tesla-m².

The results of this section are summarized in Table 3 . Specifications both before and after open-loop compensation are stated. Clearly, it is desirable to have insertion devices whose first- and second-field integrals do not require compensation. This may not be realistic, because it is extremely difficult to measure the integrals of $B_{x}$ in the presence of large $B_{y}$.

Table 3

First- and Second-Field Integral Specification.

\begin{tabular}{|c|c|c|}
\hline Quantity & Before Compensation & After Compensation \\
\hline$I 1_{x, y}$ & 100 Gauss-cm & 10 Gauss-cm \\
\hline$I 2_{x, y}$ & 100,0000 Gauss-cm & 10,000 Gauss-cm \\
\hline
\end{tabular}

\section{B. Good Field Region}

The values in Table 3 give the allowable amount of variation with gap of field integrals for particles moving along the geometric axis of the device. An equally important concept is the allowable variation in field integral over a "good field region" at fixed gap. A reasonable "good field region" is $\pm 5 \mathrm{~mm}$ horizontally, and $\pm 2 \mathrm{~mm}$ vertically. Considering that the horizontal and vertical beam sizes are $\sigma_{x}=330 \mu m$ and $\sigma_{y}=89 \mu \mathrm{m}$, respectively, this allows for $10 \sigma_{x, y}$ plus a missteering allowance of $2 \mathrm{~mm}$ horizontally and $1.1 \mathrm{~mm}$ vertically.

If one could specify that the first field integral not change by more than 10 Gauss-cm over this region, and that the second field integral not change by more than 10,000 Gauss- $\mathrm{cm}^{2}$, it would be insured that the compensation would not have to be modified when orbit changes are made. This represents the ideal situation, because it is extremely difficult to construct such a device, or even to confidently measure it without using beam.

Since insertion device photons will be accurately aligned relative to beamline $x$-ray beam position monitors and held fixed with a high degree of reproducibility from fill to fill, one can specify a more relaxed tolerance, and perform careful first- and second-field integral compensation vs. gap for a signal trajectory through the device. For the APS, the tolerances will be fixed at 100 Gauss-cm and 100,000 Gauss- $\mathrm{cm}^{2}$ across $\pm 5 \mathrm{~mm}$ horizontally and $\pm 2 \mathrm{~mm}$ vertically, including variation with gap. In terms of the normal 
$\left(b_{n}\right)$ and skew $\left(a_{n}\right)$ multipole coefficients defined at APS:

$$
\int\left(B_{y}+i B_{x}\right) d l=B_{0} L \sum_{n=0}^{\infty}\left(b_{n}+i a_{n}\right)(x+i y)^{n} .
$$

These requirements translate to $B_{0} L b_{n}=100$ Gauss-cm, 200 Gauss, 400 Gauss $/ \mathrm{cm}$, and 800 Gauss $/ \mathrm{cm}^{2}$ for $\mathrm{n}=0$, $1,2,3$, respectively. These tolerances should be compared with the lattice magnet tolerances and the effective strength of the ID itself. For example, the tolerances on the quadrupole magnets at APS are $B_{0} L b_{n}=58$ Gauss, 12 Gauss/cm, and 6 Gauss $/ \mathrm{cm}^{2}$ for $\mathrm{n}=1,2,3$, respectively. Recalling the effective strength of the ID is $B_{0} L b_{1}=242$ Gauss and $B_{0} L b_{3}=600 \mathrm{Gauss} / \mathrm{cm}^{2}$, we can set reasonable tolerances as $B_{0} L b_{n}=100$ Gauss-cm, 50 Gauss, 200 Gauss $/ \mathrm{cm}$, and 600 Gauss $/ \mathrm{cm}^{2}$. At this stage, the tolerances on skew components can be the same.

This requirement places bounds on allowable multipole components which are necessary, but not sufficient.

\section{Multipole Tolerances}

In the previous section we considered the constraints on the field quality based on beam position stability. The other requirements listed in Table 1 will also limit the multipole tolerances. These tolerances should be compared with the allowable multipole components specified in the previous section.

Since the lattice perturbation from the effective quadrupole strength of the ID is negligible, $B_{0} L b_{1}=50$ Gauss is acceptable.

The requirement on the vertical beam size is that the change of beam size should be less than $5 \%$ of the natural beam size, assuming $10 \%$ emittance coupling from the lattice magnets. The corresponding skew quadrupole strength is $B_{0} L a_{1}=157$ Gauss. Thus $B_{0} L a_{1}=50$ Gauss specified in the previous section also satisfies this requirement.

The design beam lifetime at the APS is 10 hours. The study [3] showed that the required vertical aperture is 3.3 $\mathrm{mm}$ during nominal operation. Even though the vertical vacuum chamber size (half gap of the ID chamber) is 4 $\mathrm{mm}$, a particle with horizontal oscillation amplitude of 5 $\mathrm{mm}$ should be stable within the required vertical aperture of $3.3 \mathrm{~mm}$.

In order to determine the physical aperture including the integrated multipole components in the ID, a tracking study was done. We first studied the effects on the physical aperture due to the individual multipole components, whose strengths are specified in the previous section. The result obtained by using the program RACETRACK [4] is shown in Fig. 1. In the tracking studies, we assume 34 IDs installed in the ring and consider the particle lost if its amplitude is larger than $4 \mathrm{~mm}$ at the ID section. From the figure we find that we need to tighten the skew multipole tolerances. The skew multipoles have more effects on reducing the physical aperture because
Physical Aperture

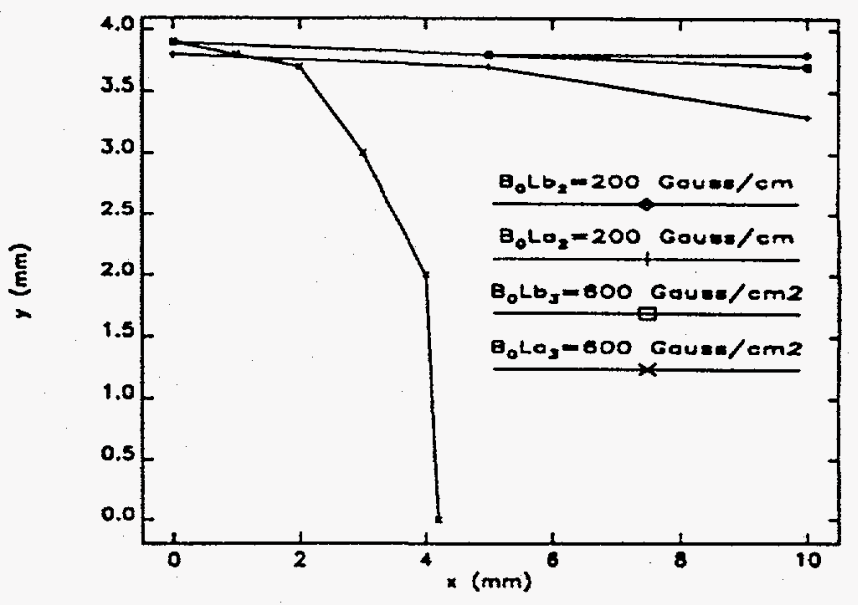

Figure 1

Physical Aperture with Multipole Component.

the lattice tunes are close to the sum- and/or differenceresonance line caused by the skew multipoles. After a few iterations we found that the aperture requirement can be met at $B_{0} L b_{2}=200 \mathrm{Gauss} / \mathrm{cm}, B_{0} L a_{2}=100 \mathrm{Gauss} / \mathrm{cm}$, $B_{0} L b_{3}=300$ Gauss $/ \mathrm{cm}^{2}$, and $B_{0} L a_{3}=50$ Gauss $/ \mathrm{cm}^{2}$. Taking into account all effects, i.e. tune shift, beam size change, dynamic aperture, and beam position stability, we arrive at the reasonably consistent set of multipole tolerances shown in Table 4.

Table 4

ID Integrated Multipole Tolerance Specifications.

\begin{tabular}{|c|c|c|}
\hline $\mathrm{n}$ & $\begin{array}{c}\text { Normal Component } \\
B_{0} L b_{n}\end{array}$ & $\begin{array}{c}\text { Skew Component } \\
B_{0} L a_{n}\end{array}$ \\
\hline 0 & $100 \mathrm{Gauss}-\mathrm{cm}$ & $100 \mathrm{Gauss}-\mathrm{cm}$ \\
\hline 1 & $50 \mathrm{Gauss}$ & $50 \mathrm{Gauss}$ \\
\hline 2 & $200 \mathrm{Gauss} / \mathrm{cm}$ & $100 \mathrm{Gauss} / \mathrm{cm}$ \\
\hline 3 & $300 \mathrm{Gauss} / \mathrm{cm}^{2}$ & $50 \mathrm{Gauss} / \mathrm{cm}^{2}$ \\
\hline
\end{tabular}

A conclusion is that, if the requirements on the firstand second-field integrals are satisfied, the requirement on the multipole tolerance is also satisfied.

\section{REFERENCES}

[1] L. Smith, "Effects of Wigglers and Undulators on Beam Dynamics," LBL ESG TECH Note-24 (1986).

[2] Y.-C. Chae and E. A. Crosbie, "Tracking Studies of Insertion Device Effects on Dynamic Aperture in the APS Storage Ring," Proc. of the 1993 Part. Accel. Conf., Washington, D. C. (1993), also in ANL Report LS-196 (1992).

[3] H. M. Bizek, private communication, (1994).

[4] F. Iazzourene, et al., "RACETRACK User's Guide, Version 4," Sincrotrone Trieste ST/M-92/7 (1992). 